\title{
High-resolution Three-dimensional Surface Imaging Microscope Based on Digital Fringe Projection Technique
}

\author{
Cheng-Yang Liu, Tzu-Ping Yen, Chien-Wen Chen \\ Department of Biomedical Engineering, National Yang-Ming University, Taipei City, Taiwan, cyliu66@ym.edu.tw
}

\begin{abstract}
The three-dimensional (3-D) micro-scale surface imaging system based on the digital fringe projection technique for the assessments of microfiber and metric screw is presented in this paper. The proposed system comprises a digital light processing (DLP) projector, a set of optical lenses, a microscope, and a charge coupled device (CCD). The digital seven-step fringe patterns from the DLP projector pass through a set of optical lenses before being focused on the target surface. A set of optical lenses is designed for adjustment and size coupling of fringe patterns. A high-resolution CCD camera is employed to picture these distorted fringe patterns. The wrapped phase map is calculated by seven-step phase-shifting calculation from these distorted fringe patterns. The unwrapping calculation with quality guided path is introduced to compute the absolute phase values. The dimensional calibration methods are used to acquire the transformation between real 3-D shape and the absolute phase value. The capability of complex surface measurement for our system is demonstrated by using ISO standard screw M1.6. The experimental results for microfiber with $3 \mu \mathrm{m}$ diameter indicate that the spatial and vertical resolutions can reach about $3 \mu \mathrm{m}$ in our system. The proposed system provides a fast digital imaging system to examine the surface features with high-resolution for automatic optical inspection industry.
\end{abstract}

Keywords: Digital imaging, fringe projection, micro-scale measurement.

\section{INTRODUCTION}

The scale of microelectronic components continues to decrease with the rapid development of semiconductor technology. Three-dimensional (3-D) shape measurement techniques have played an important role for manufacturers in engineering fields. The traditional contact measurements have to touch the target surface in order to determine the dimensions of targets [1]-[3]. The targets could be damaged when the contact measurement physically touches the target surface. Measurement errors can also be caused by vibrations when targets are measured by contact measurement. Therefore, there is a need to improve the speed and resolution for 3-D shape measurements. Recently, non-contact optical inspection techniques have been established for 3-D shapes and complex geometry measurements [4]-[16]. The advantages of non-contact optical inspection include high measurement speed, quick and easy operations, and the ability to achieve full-field surface measurement. The noncontact optical inspection can be applied in many areas such as the manufacturing process of products, physiological and behavior characteristics of biometrics, 3-D shape measurement of micro-electro-mechanical components, machine vision, and reverse engineering.

In computer technology and industrial manufacturing, digital fringe projection and multi-step phase-shifting techniques have been widely used in optical 3-D surface measurements. All kinds of optical imaging methods using fringe projection for 3-D surface measurements have been proposed and investigated [17]-[34]. These optical systems generally use a projector to screen fringe patterns onto the surface of objects, a charge coupled device (CCD) camera to catch these deformed fringe patterns, and an industrial computer to estimate phase values and reconstruction of shape geometry. The operational processes of 3-D shape reconstruction technique comprise digital fringe projection, phase-shifting calculation, and the conversion scheme method for phase to real size. A variety of phase-shifting algorithms have been proposed in many references [35]-[42]. In the digital fringe projection technique, a series of sinusoidal fringe patterns are calculated and created by computer. These digital fringe patterns are projected onto the object surface by using the digital light processing (DLP) projector. The wrapped phase maps are acquired by general phase-shifting calculation. The unwrapped phase maps are obtained by various phase unwrapping calculations. The unwrapping calculation with quality guided path has been recommended to diminish the phase error in varied fringe patterns [35]. Finally, the 3-D shape geometry is reproduced from unwrapped phase maps. The geometric relationship between phase and real size is obtained by dimensional conversion. In this study, the high-resolution micro-scale surface imaging system (MSIS) based on digital phase- 
shifting technique is presented. The principle of MSIS is defined in Section 2. The measurement results of 3-D shape of targets are illustrated in Section 3. The summary is presented in Section 4.

\section{PRINCIPLE OF MICRO-SCALE SURFACE IMAGING SYSTEM}

In order to obtain the 3-D shape of micro-scale targets, the scale of digital fringe patterns needs to be decreased. Fig.1. shows the components of the MSIS that consists of a DLP projector (Acer K132+), a digital camera (Tucsen TrueChrome II) with $1920 \times 1080$ resolution, a set of optical lenses, a stereo-microscope (Leica M50), and an industrial computer. The set of optical lenses includes a biconvex lens (SLB-80B-100P), a biconcave lens (SLB-50B-50N), and a plano-convex lens (SLB-80-100P). The dimensions of these lenses can be referred to the part number from the Sigmakoki Company. The relationship of the position with the set of optical lenses is obtained by ray tracing calculation. Fig.1.c) describes the ray tracing result of the set of optical lenses. A set of optical lenses is used to decrease the pattern size for light adjustment and coupling. The biconvex lens is employed to decrease the image size of fringe patterns from the DLP projector and the biconcave lens is adopted as a collimated image converter. The image size of fringe patterns is adjusted by the plano-convex lens for passing into a $\mathrm{C}$-mount adapter. Then, the fringe patterns are straightened by a stereomicroscope and projected onto the surface of micro-scale targets. Finally, these deformed fringe patterns are received again by a stereo-microscope and captured by a digital camera. The incidence and reflection of optical propagation are almost normal to the target surface.

Fig.2. depicts the circuit of 3-D surface imaging acquisition and processing. The numerical and calculation processes of the MSIS are briefly described here. The digital fringe patterns are generated and manipulated precisely by an industrial computer. The advantages of digital fringe projection are full-field inspection, fast data processing, highresolution and non-contact measurement. The phase maps are derived from the fringe patterns via phase-shifting algorithm [35]-[42]. The mathematical concept and computing process of the phase-shifting algorithm are briefly represented here. A fringe pattern for intensity distribution is described as: $I_{N}=$ $I_{g}(i, j)+I_{m}(i, j) \times \cos [\phi(i, j)+\beta]$. The pixel positions along horizontal and vertical orientations in the patterns are $i$ and $j$, respectively. The intensity distribution of the $N$ th pattern at the pixel position is $I_{N}(i, j)$. The average intensity of the background illumination is $I_{g}(i, j)$. The distributed modulation of the pattern intensity is $I_{m}(i, j) . \phi(i, j)$ is the phase value that correlates closely with profile data of a target. The phase-shift of the fringe pattern is $\beta$. The multi-step phase-shifting technique is obtained from the well-known three-step phaseshifting calculation. Because the profile smoothness can be significantly improved by using the seven-step phase-shifting calculation [27], the seven-step phase-shifting calculation is used to achieve high-resolution 3-D surface imaging. The seven intensity distributions of the phase-shifting calculation can be expressed as:

$$
\begin{gathered}
I_{1}=I_{g}+I_{m} \times \cos [\phi(i, j)-3 \pi / 2] \\
I_{2}=I_{g}+I_{m} \times \cos [\phi(i, j)-\pi] \\
I_{3}=I_{g}+I_{m} \times \cos [\phi(i, j)-\pi / 2] \\
I_{4}=I_{g}+I_{m} \times \cos [\phi(i, j)] \\
I_{5}=I_{g}+I_{m} \times \cos [\phi(i, j)+\pi / 2] \\
I_{6}=I_{g}+I_{m} \times \cos [\phi(i, j)+\pi] \\
I_{7}=I_{g}+I_{m} \times \cos [\phi(i, j)+3 \pi / 2]
\end{gathered}
$$

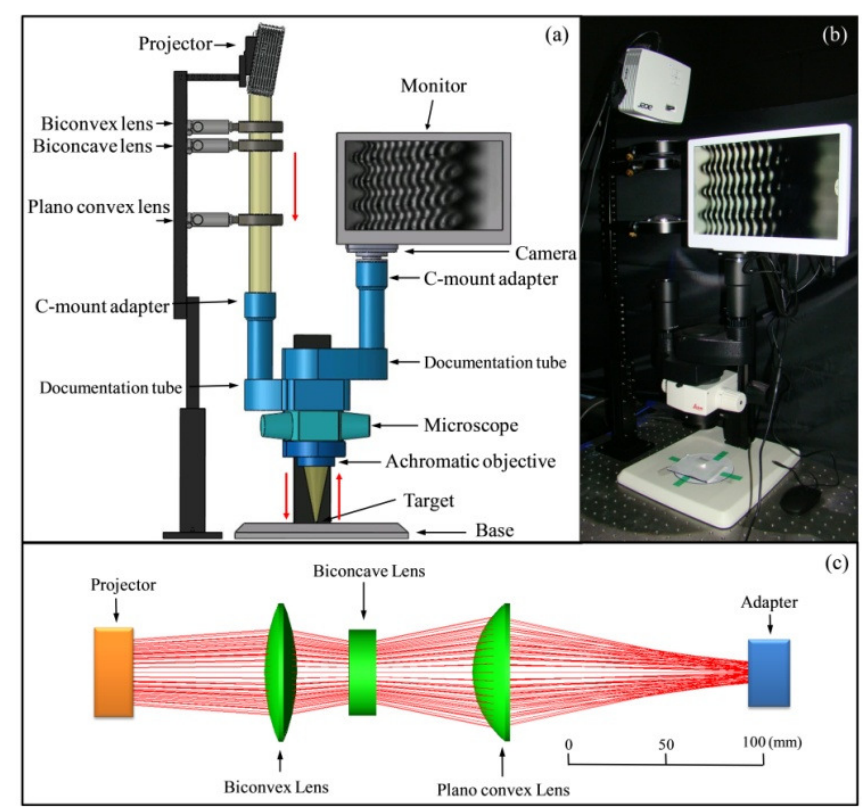

Fig.1. Micro-scale surface imaging system: a) schematic diagram and b) picture. c) Ray tracing result of optical lenses for light adjustment and pattern size coupling. The red arrows in a) indicate the direction of optical propagation.

Fringe patterns generated by program

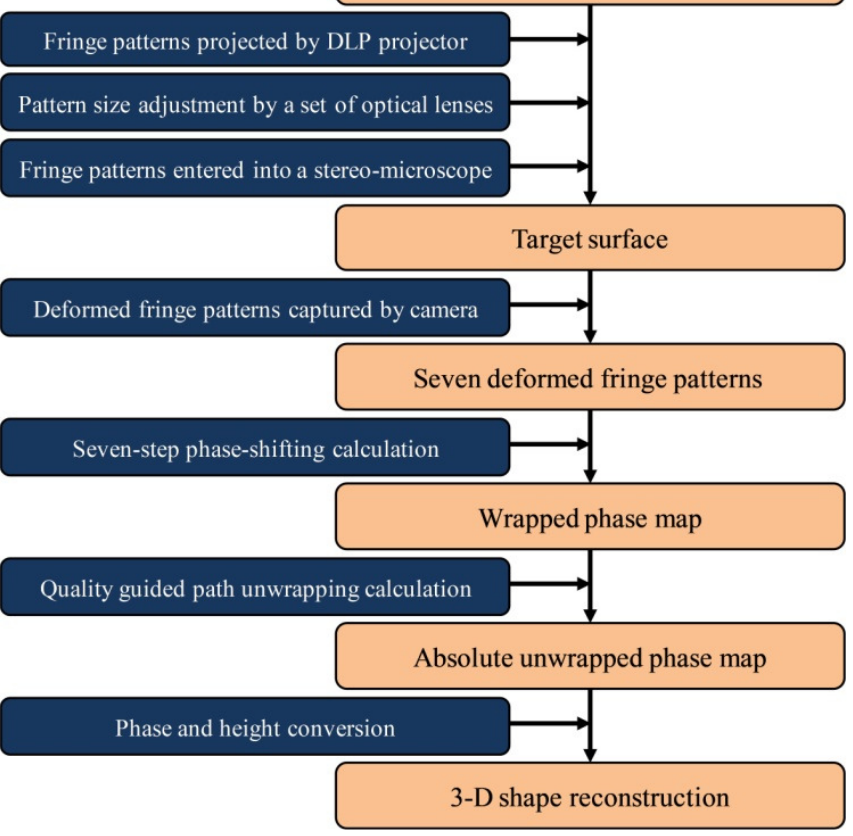

Fig.2. Circuit of 3-D surface imaging acquisition and processing. 
The wrapped phase maps for the target plane are computed by applying the seven-step phase-shifting calculation. The difference in intensity between the fringe adjustment and the light source is ignored in (1). The phase function $\phi(i, j)$ is extracted as: $\phi(i, j)=\tan ^{-1}\left[\left(3 I_{3}+I_{7}-I_{1}-3 I_{5}\right) /\left(4 I_{4}-2 I_{2}-2 I_{6}\right)\right]$. The values of wrapped phase $\phi(i, j)$ are in a module of $2 \pi$ and the differential phase angle between two consecutive fringes is in multiples of $2 \pi$. An effective method in phase unwrapping is required to eliminate unreliable values. The quality guided path calculation has been proposed to unwrap the image pixels with highest reliability [35]. Therefore, we employ the quality guided path calculation to demodulate wrapped phase. The phase errors formed by discontinuous surfaces are easily eliminated in phase-unwrapping calculation. The second difference in the quality guided path calculation is used to specify the reliability of a pixel. The path of the unwrapping process is decided by the pixel reliability. The error propagation can be prevented by evaluating pixel quality with reliability. The second difference computed pixel by pixel is $D$ and the pixel reliability is described as $R=1 / D$. When the $D$ value is small, the pixel in the phase map is reliable. An edge is constructed from each pixel with its ambient pixel. A final summation of the reliabilities for neighboring pixels is the reliability of an edge. We clarify an unwrapping path on the reliability value of each edge. The edge with high reliability value is calculated first and categorized in terms of reliability. The values of absolute phase are received along a discontinuous route. In order to reconstruct actual 3-D surface, the relationship between real size and absolute phase must be corrected in the computational process. The surface shape of object can be quantified by the triangulation method [37]. The pixel height $h$ in the phase map is expressed as $h(i, j)=P$ $\phi(i, j) / 2 \pi \tan \theta$. The fringe pitch in a pattern is $P$, the incident angle of illumination is $\theta$.

The configuration of MSIS is associated with the coefficients in the pixel height. The realizable optical resolution of MSIS decides on the pitch of fringe pattern and the step of phase-shift. The pixel height is estimated from the phase function. The pitch of fringe pattern is modified by using projector and calculating code. The commercial gauge block (Mitutoyo 170383) is employed for testing the conversion of real height and phase. The dimensions of the standard gauge block are $28 \mathrm{~mm} \times 8 \mathrm{~mm} \times 0.5 \mathrm{~mm}$. The standard gauge block is located on a chosen reference plane. The relationship between phase and real height is calculated by the phase function and the fringe pitch. In this study, the fringe pitch $P$ is $150 \mu \mathrm{m}$ and the measurement resolution of MSIS is predicted in $3 \mu \mathrm{m}$. Because the behavior of fringe patterns is quite responsive to the stray light from the surrounding environment, the MSIS is positioned inside a darkroom. The darkroom is used to control diffused light and avoid any influence of the environment.

\section{EXPERIMENTAL RESULTS}

The screw shape is usually inspected by contact measurements in a traditional fabrication process, however, the measurement deviations are easily caused by man-made influences. Furthermore, a screw with a small diameter is very difficult to gauge by current methods. In order to evaluate the imaging quality of MSIS, the metric screws of the International Organization for Standardization (ISO) are chosen for the measurements. The design rules of metric screws are clarified in standard ISO 68-1. The M series of screws comprises a symmetric V-shape. The material of the standard screw is stainless steel. The diameter and pitch of the standard M1.6 screw are $1.6 \mathrm{~mm}$ and $0.35 \mathrm{~mm}$. The 3-D shape reconstructions for ISO standard screws are described in this section. Fig.3. depicts the seven fringe patterns projected on the standard screw M1.6. The picture in the bottom right of Fig.3. is the M1.6 screw picture. The fringe pitch is $150 \mu \mathrm{m}$ and the phase shift in seven fringe patterns is $\pi / 2$. The seven-step phase-shifting calculation is performed on these seven deformed fringe patterns.
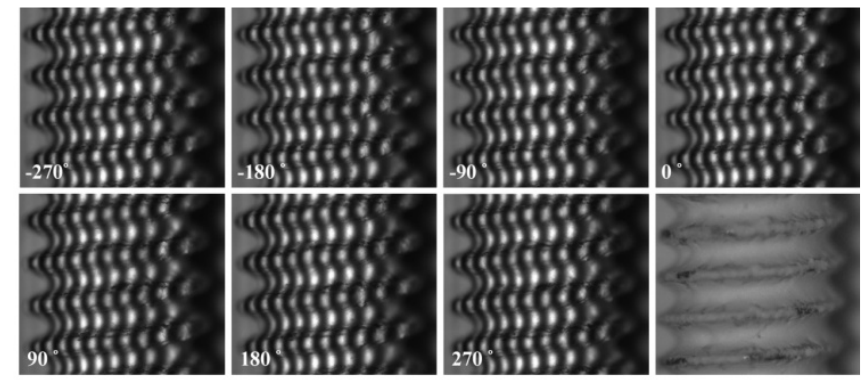

Fig.3. Seven fringe patterns projected on the standard screw M1.6. The picture in the bottom right is the picture of standard screw M1.6.

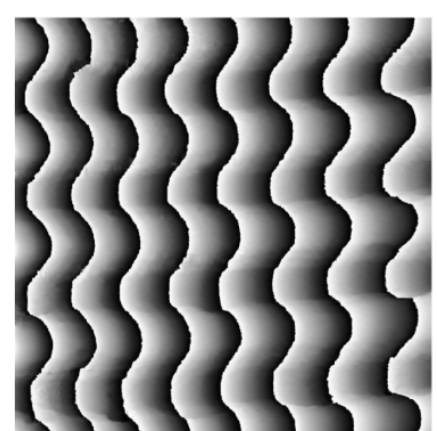

(a)

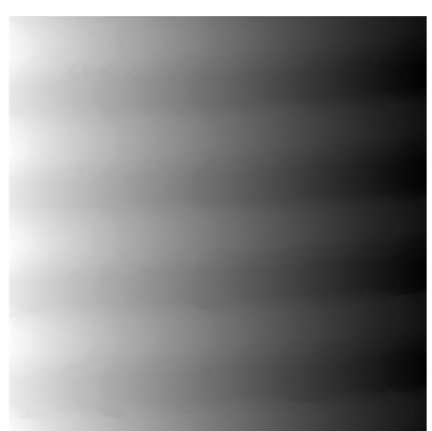

(b)
Fig.4. Calculated a) wrapped phase map and b) unwrapped phase map by seven-step phase-shifting and quality guided path unwrapping for the standard screw M1.6.

Fig.4.a) depicts the wrapped phase map for the standard screw M1.6. It is shown that the change in screw profile especially impacts the wrapped phase map. In Fig.4.a), the phase values are a periodic function of $2 \pi$. We apply quality guided path calculation to receive the unwrapped phase values. Fig.4.b) illustrates the unwrapped phase map for the standard screw M1.6. The unwrapped phase values are the serial growing numbers in which the highest number exhibits a white symbol and the lowest number exhibits a black symbol. The extracted unwrapped phase map indicates screw characteristics which are distinctly exterminating error propagation in the quality guided path calculation. 


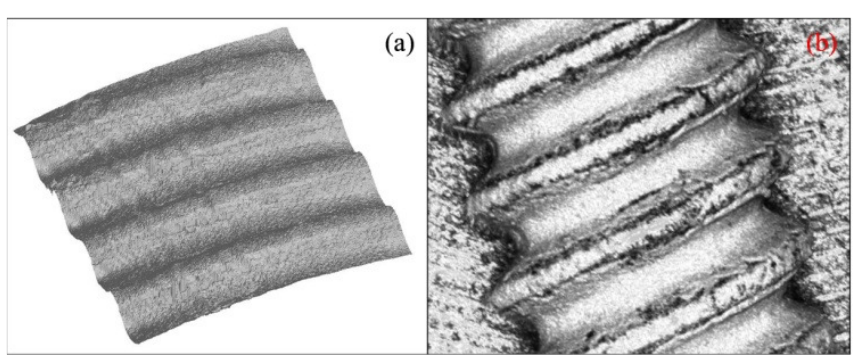

Fig.5. 3-D shape reconstruction of the standard screw M1.6 captured by a) MSIS and b) LSCM.

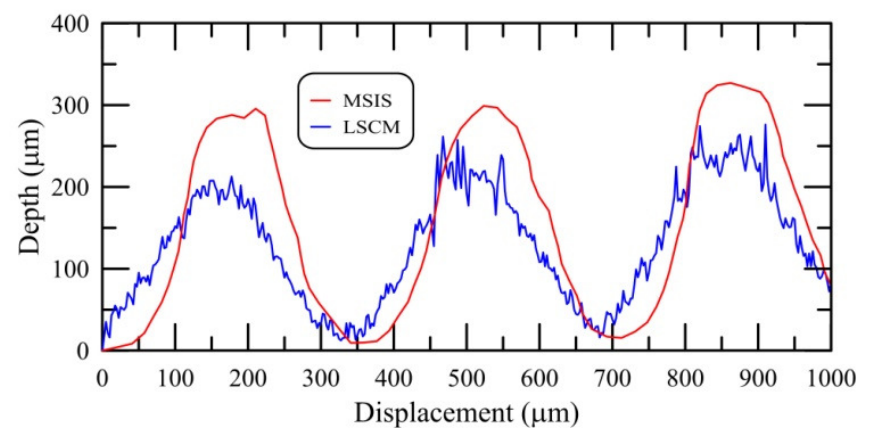

Fig.6. Cross-section profiles of the standard screw M1.6 in MSIS and LSCM.

The reference plane reduction method is employed to eliminate the background perturbation in the unwrapped phase values [27]. In order to obtain the reference plane, the perfect fringe patterns are projected onto a blank plane from a projector. The calculations in quality guided path unwrapping and seven-step phase-shifting are also applied to compute the unwrapped phase value for the reference plane. Therefore, the absolute phase value without background noise is achieved by deducting the unwrapped phase value of a reference plane from the unwrapped phase value of a screw. The actual 3-D profile information is obtained by using calibrated relationship from the absolute phase map. A commercial laser scanning confocal microscopy (LSCM, LEXT OLS4100) is used to compare with the proposed system. Fig.5., depicts the 3-D reconstruction of the standard screw M1.6 captured by MSIS and LSCM. It is shown that the complex surface of the standard screw can be reconstructed by the proposed MSIS. The LSCM produces measurement results with clearly noticeable ripples and defects. The imaging error in the LSCM is larger than in the MSIS. In order to compare the imaging differences between the MSIS and LSCM, the cross-section profiles of the standard screw M1.6 by MSIS and LSCM are illustrated in Fig.6. We can see that the LSCM produces stronger lowfrequency corrugations than the MSIS, and the MSIS generates a significant profile in which the ripples are hardly noticeable. The seven-step phase-shifting method obviously mended the smoothness of the screw surface. The nonlinear phase errors are compensated by quality guided path unwrapping.

In order to demonstrate the imaging resolution of the MSIS, the microfiber with $3 \mu \mathrm{m}$ diameter is used for measurements. The material of the microfiber is undoped silica. Fig.7. depicts the seven fringe patterns projected on the $3 \mu \mathrm{m}$ microfiber. The picture in the bottom right is the microscope image of $3 \mu \mathrm{m}$ microfiber. According to the quality guided path unwrapping and the seven-step phase-shifting calculations, the wrapped phase map and unwrapped phase map for microfiber are obtained and illustrated in Fig.8. It can be seen that the shape of the microfiber is clearly identified in both phase maps. Fig.9. depicts the 3-D reconstruction of the $3 \mu \mathrm{m}$ microfiber captured by MSIS and LSCM. When the fringe patterns are applied, almost no regular ripples are noticeable in the imaging results of MSIS. The LSCM generates a 3-D reconstruction with random noise that is hardly comparable with results of MSIS due to the noise encoded in the shape. To further validate the facility of MSIS, the cross-section profiles of microfiber are also measured.

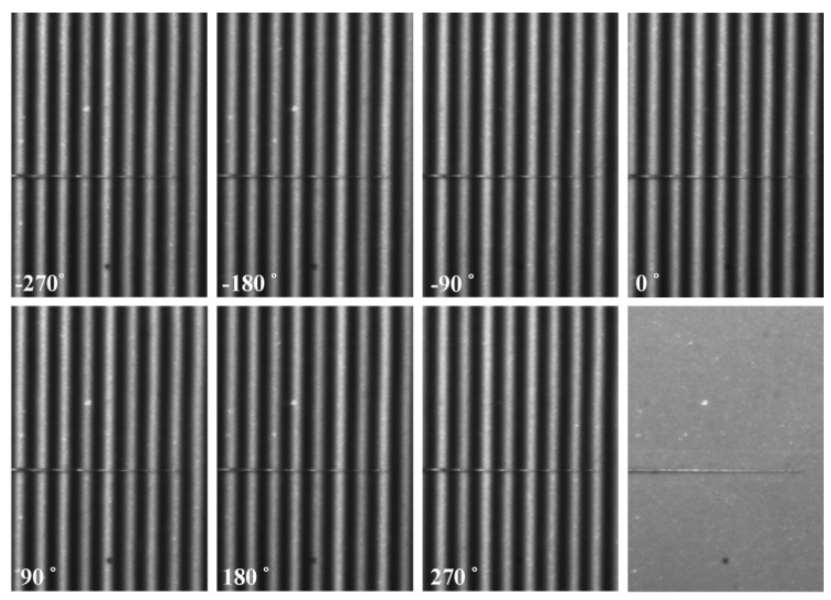

Fig.7. Seven fringe patterns projected on the $3 \mu \mathrm{m}$ microfiber. The picture in the bottom right is the microscope image of $3 \mu \mathrm{m}$ microfiber.

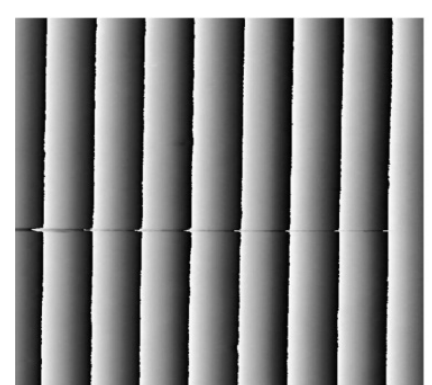

(a)

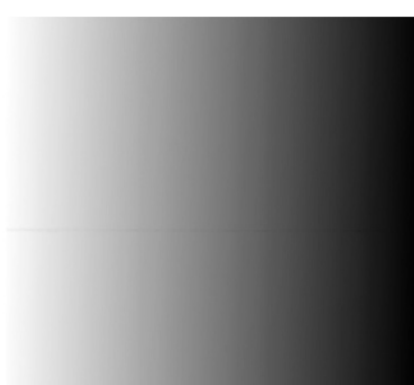

(b)
Fig.8. Calculated a) wrapped phase map and b) unwrapped phase map by seven-step phase-shifting and quality guided path unwrapping for the $3 \mu \mathrm{m}$ microfiber.

Fig.10. depicts the cross-section profiles of the $3 \mu \mathrm{m}$ microfiber in MSIS and LSCM. The ideal circle in Fig.10. is used to contrast with the imaging profiles of MSIS and LSCM. The roundness values for MSIS and LSCM are 0.5 and 1.75, respectively. The imaging results show that 3-D shape of microfiber is faithfully reconstructed in smooth surfaces without spurious features. The quality guided path unwrapping and the seven-step phase-shifting calculations avoid the effect of phase noise. The proposed MSIS is better than LSCM in terms of measurement accuracy. Besides, 
LSCM can only measure one side profile of the microfiber. The MSIS can fully achieve an accurate 3-D measurement with a rather small level. The measurement capabilities of complexity and high-resolution are well-proven for MSIS.

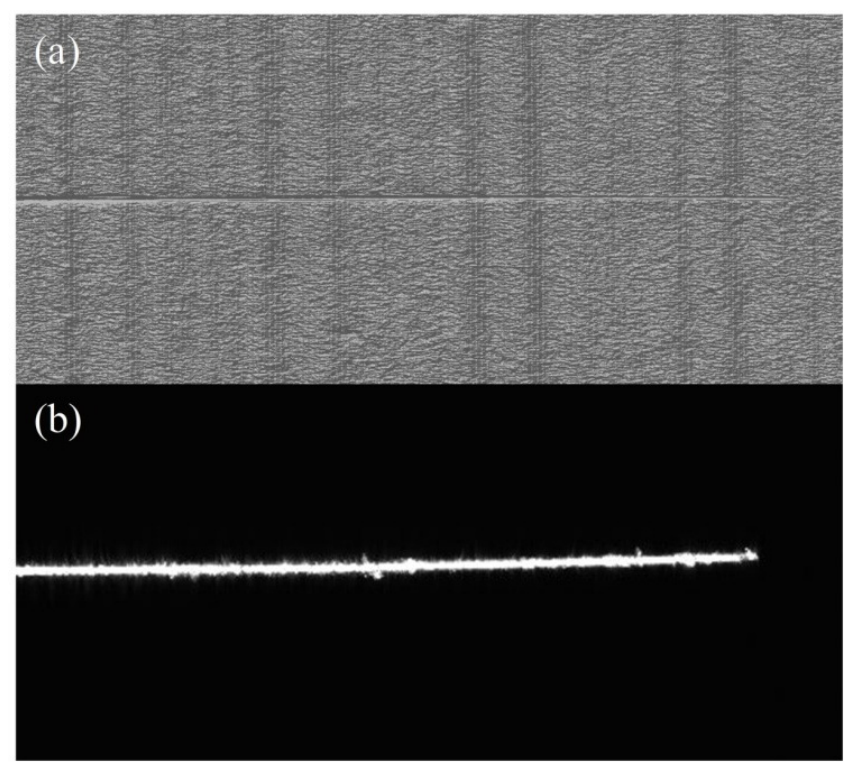

Fig.9. 3-D shape reconstruction of the $3 \mu \mathrm{m}$ microfiber captured by a) MSIS and b) LSCM.

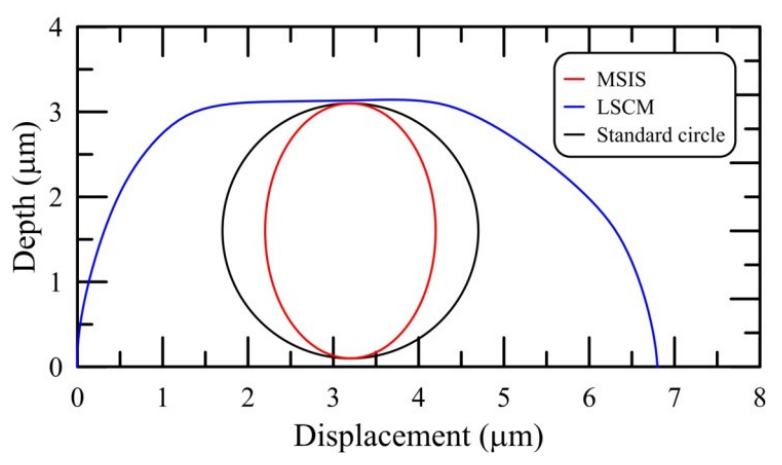

Fig.10. Cross-section profiles of the $3 \mu \mathrm{m}$ microfiber in MSIS and LSCM.

\section{CONCLUSIONS}

This paper has presented the MSIS based on the digital fringe projection technique to reduce phase error and create high-quality imaging in the 3-D shape measurements for the microfiber and metric screw. The proposed MSIS comprises a DLP projector, a set of optical lenses, a microscope, and a CCD. Compared to the LSCM, the MSIS is not influenced by the surface reflectivity and ambient light. We have analyzed the imaging quality of MSIS and LSCM for the microfiber and metric screw. The MSIS is more suitable for 3-D shape measurement of micro-scale targets. The imaging results have verified the validity of the MSIS, showing that the phase error is negligible and it can generate high-quality imaging. The imaging resolution of the MSIS can reach about $3 \mu \mathrm{m}$. The performance of MSIS is not at the cost of reducing the measurement speed or involving further post-processing. Therefore, the MSIS is very beneficial for real-time micro- scale 3-D shape inspection in a time critical condition. This subject is considered in our further research.

\section{ACKNOWLEDGMENT}

The authors would like to thank the Ministry of Science and Technology (MOST) of Taiwan and Yen Tjing Ling Medical Foundation to sponsor this study. The grant numbers are MOST 108-2221-E-010-012-MY3, MOST 109-2923-E-010001-MY2 and CI-109-24.

\section{REFERENCES}

[1] Yip, F., Venart, J. (1971). An elastic analysis of deformation of rough spheres, rough cylinders and rough annuli in contact. Journal of Physics D: Applied Physics, 4, 1470-1486.

[2] Kato, S., Yamaguchi, K., Kato, T. (1981). A method to measure contact pressure between metallic surfaces by changes in surface roughness. Journal of Engineering for Industry, 103, 210-217.

[3] Jang, Y. (2000). Transient thermoelastic contact problems for an elastic foundation. International Journal of Solids and Structures, 37, 1997-2004.

[4] Chen, F., Brown, G., Song, M. (2000). Overview of three-dimensional shape measurement using optical methods. Optical Engineering, 39, 10-22.

[5] Reich, C., Ritter, R., Thesing, J. (2000). 3-D shape measurement of complex objects by combining photogrammetry and fringe projection. Optical Engineering, 39, 224-231.

[6] Sitnik, R., Kujawinska, M., Woznicki, J. (2002). Digital fringe projection system for large-volume 360-deg shape measurement. Optical Engineering, 41, 443-449.

[7] Zhang, S., Huang, P. (2006). High-resolution, real-time three-dimensional shape measurement. Optical Engineering, 45, 123601.

[8] Du, H., Wang, Z. (2007). Three-dimensional shape measurement with an arbitrarily arranged fringe projection profilometry system. Optics Letters, 32, 2438-2440.

[9] Park, S., Chang, M. (2009). Reverse engineering with a structured light system. Computers \& Industrial Engineering, 57, 1377-1384.

[10] Hong, D., Lee, H., Kim, M., Cho, H., Moon, J. (2009). Sensor fusion of phase measuring profilometry and stereo vision for three-dimensional inspection of electronic components assembled on printed circuit boards. Applied Optics, 48, 4158-4169.

[11] Wang, Z., Du, H., Park, S., Xie, H. (2009). Threedimensional shape measurement with a fast and accurate approach. Applied Optics, 48, 1052-1061.

[12] Liu, K., Wang, Y., Lau, D., Hao, Q., Hassebrook, L. (2010). Dual-frequency pattern scheme for high-speed 3-D shape measurement. Optics Express, 18, 52295244.

[13] Su, X., Zhang, Q. (2010). Dynamic 3-D shape measurement method: a review. Optics and Lasers in Engineering, 48, 191-204.

[14] Geng, J. (2011). Structured-light 3D surface imaging: a tutorial. Advances in Optics and Photonics, 3, 128-160. 
[15] Zuo, C., Chen, Q., Gu, G., Feng, S., Feng, F. (2012). High-speed three-dimensional profilometry for multiple objects with complex shapes. Optics Express, 20, 19493-19510.

[16] Ma, S., Quan, C., Zhu, R., Chen, L., Li, B., Tay, C. (2012). A fast and accurate gamma correction based on Fourier spectrum analysis for digital fringe projection profilometry. Optics Communications, 285, 533-538.

[17] Brakhage, P., Notni, G., Kowarschik, R. (2004). Image aberrations in optical three-dimensional measurement systems with fringe projection. Applied Optics, 43, 3217-3223.

[18] Marklund, O., Huntley, J., Cusack, R. (2007). Robust unwrapping algorithm for three-dimensional phase volumes of arbitrary shape containing knotted phase singularity loops. Optical Engineering, 46, 085601.

[19] Zhang, S., Yau, S. (2007). Generic nonsinusoidal phase error correction for three-dimensional shape measurement using a digital video projector. Applied Optics, 46, 36-43.

[20] Li, Z., Shi, Y., Wang, C., Wang, Y. (2008). Accurate calibration method for a structured light system. Optical Engineering, 47, 053604.

[21] Pan, B., Kemao, Q., Huang, L., Asundi, A. (2009). Phase error analysis and compensation for nonsinuoidal waveforms in phase-shifting digital fringe projection profilometry. Optics Letters, 34, 416-418.

[22] Fu, Y. Luo, Q. (2011). Fringe projection profilometry based on a novel phase shift method. Optics Express, 19, 21739-21747.

[23] Zuo, C., Chen, Q., Gu, G., Feng, S., Feng, F., Li, R., Shen, G. (2013). High-speed three-dimensional shape measurement for dynamic scenes using bi-frequency tripolar pulse-width-modulation fringe projection. Optics and Lasers in Engineering, 51, 953-960.

[24] Yin, Y., Wang, M., Gao, B., Liu, X., Peng, X. (2015). Fringe projection 3D microscopy with the general imaging model. Optics Express, 23, 6846-6857.

[25] Li, B., Zhang, S. (2015). Flexible calibration method for microscopic structured light system using telecentric lens. Optics Express, 23, 25795-25803.

[26] Babaie, G., Abolbashari, M., Farahi, F. (2015). Dynamics range enhancement in digital fringe projection technique. Precision Engineering, 39, 243251.

[27] Liu, C., Yen, T. (2016). Digital multi-step phaseshifting profilometry for three-dimensional ballscrew surface imaging. Optics \& Laser Technology, 79, 115123.

[28] Hu, Y., Chen, Q., Tao, T., Li, H., Zuo, C. (2017). Absolute three-dimensional micro surface profile measurement based on a Greenough-type stereomicroscope. Measurement Science and Technology, 28, 045004.

[29] Wang, M., Yin, Y., Deng, D., Meng, X., Liu, X., Peng, X. (2017). Improved performance of multi-view fringe projection 3D microscopy. Optics Express, 25, 1940819421.
[30] Pistellato, M., Bergamasco, F., Albarelli, A., Cosmo, L., Gasparetto, A., Torsello, A. (2019). Robust phase unwrapping by probabilistic consensus. Optics and Lasers in Engineering, 121, 428-440.

[31] Wu, G., Wu, Y., Li, L., Liu, F. (2019). High-resolution few-pattern method for 3D optical measurement. Optics Letters, 44, 3602-3605.

[32] Gai, S., Da, F., Tang, M. (2019). A flexible multi-view calibration and 3D measurement method based on digital fringe projection. Measurement Science and Technology, 30, 025203.

[33] Xu, Y., Zhao, H., Jiang, H., Li, X. (2019). Highaccuracy 3D shape measurement of translucent objects by fringe projection profilometry. Optics Express, 27, 18421-18434.

[34] Hu, Y., Chen, Q., Feng, S., Tao, T., Asundi, A., Zuo, C. (2019). A new microscopic telecentric stereo vision system - Calibration, rectification, and threedimensional reconstruction. Optics and Lasers in Engineering, 113, 14-22.

[35] Herráez, M., Burton, D., Lalor, M., Gdeisat, M. (2002). Fast two-dimensional phase-unwrapping algorithm based on sorting by reliability following a noncontinuous path. Applied Optics, 41, 7437-7444.

[36] Wang, Z., Du, H., Bi, H. (2006). Out-of-plane shape determination in generalized fringe projection profilometry. Optics Express, 14, 12122-12133.

[37] Zuo, C., Chen, Q., Feng, S., Feng, F., Gu, G., Sui, X. (2012). Optimized pulse width modulation pattern strategy for three-dimensional profilometry with projector defocusing. Applied Optics, 51, 4477-4490.

[38] Goldstein, G., Creath, K. (2015). Quantitative phase microscopy: automated background leveling techniques and smart temporal phase unwrapping. Applied Optics, 54, 5175-5185.

[39] Yang, T., Kim, H., Lee, K., Kim, B., Choi, Y. (2016). Single-shot and phase-shifting digital holographic microscopy using a 2-D grating. Optics Express, 24, 9480-9488.

[40] Padilla, M., Servin, M., Garnica, G. (2017). Profilometry with digital fringe-projection at the spatial and temporal Nyquist frequencies. Optics Express, 25, 22292-22302.

[41] Guo, W., Wu, Z., Xu, R., Zhang, Q., Fujigaki, M. (2019). A fast reconstruction method for threedimensional shape measurement using dual-frequency grating projection and phase-to-height lookup table. Optics \& Laser Technology, 112, 269-277.

[42] Kulkarni, R., Banoth, E., Pal, P. (2019). Automated surface feature detection using fringe projection: an autoregressive modeling-based approach. Optics and Lasers in Engineering, 121, 506-511. 